\title{
Antimicrobial Photodynamic Therapy and Dental Plaque: A Systematic Review of the Literature
}

\author{
G. C. Santin, ${ }^{1}$ D. S. B. Oliveira, ${ }^{1}$ R. Galo, ${ }^{2}$ M. C. Borsatto, ${ }^{1}$ and S. A. M. Corona ${ }^{3}$ \\ ${ }^{1}$ Department of Pediatric Clinics, Preventive and Community Dentistry, Dental School of Ribeirão Preto, University of São Paulo, \\ Ribeirão Preto, SP, Brazil \\ ${ }^{2}$ Department of Prosthesis and Dental Materials, Dental School of Araraquara, São Paulo State University, Araraquara, SP, Brazil \\ ${ }^{3}$ Department of Restorative Dentistry, Dental School of Ribeirão Preto, University of São Paulo, \\ Avenida do Café, s/n, Monte Alegre, 14040-904 Ribeirão Preto, SP, Brazil
}

Correspondence should be addressed to S. A. M. Corona; silmaracorona@forp.usp.br

Received 2 July 2014; Accepted 9 September 2014; Published 14 October 2014

Academic Editor: Toni Zeinoun

Copyright (C) 2014 G. C. Santin et al. This is an open access article distributed under the Creative Commons Attribution License, which permits unrestricted use, distribution, and reproduction in any medium, provided the original work is properly cited.

\begin{abstract}
Background. The aim of this study was to perform a systematic review of the literature on the efficacy of antimicrobial photodynamic therapy (PDTa) on cariogenic dental biofilm. Types of Studies Reviewed. Studies in vivo, in vitro, and in situ were included. Articles that did not address PDTa, those that did not involve cariogenic biofilm, those that used microorganisms in the plankton phase, and reviews were excluded. Data extraction and quality assessments were performed independently by two raters using a scale. Results. Two hundred forty articles were retrieved; only seventeen of them met the eligibility criteria and were analyzed in the present review. Considerable variability was found regarding the methodologies and application protocols for antimicrobial PDTa. Two articles reported unfavorable results. Practical Implications. The present systematic review does not allow drawing any concrete conclusions regarding the efficacy of antimicrobial PDTa, although this method seems to be a promising option.
\end{abstract}

\section{Background}

Dental caries has a multifactor etiology, including cariogenic microorganisms in the oral cavity. These microorganisms use a glycolytic pathway to produce acids that are capable of demineralizing tooth enamel and dentin. Some microorganisms use sucrose as substrate for the production of intracellular and extracellular polysaccharides, which are highly cariogenic $[1,2]$. Moreover, a large portion of periodontopathogenic bacteria is found in dental biofilm (plaque), which underscores the considerable contribution of this substance in the development of adverse health conditions of the oral cavity.

Dental biofilm is a three-dimensional structure of bacterial communities adhered to the tooth surface [3]. Microcolonies of bacterial cells account for 15 to $20 \%$ of dental biofilm and the rest is composed of exopolysaccharides, water, proteins, salts, and the cell fragments $[4,5]$. Pores or channels of water among the bacterial microcolonies serve as a primitive circulation system, allowing the passage of nutrients and other agents, which affect the distribution and movement of molecules in biofilm [3]. The constitution of biofilm protects colonizing species from adverse factors in the environment, such as defense mechanisms of the host and potentially toxic substances (lethal chemical agents and antibiotics) [4]. Moreover, slow-growing cells, which are one of the characteristics of bacteria found in deeper portions of biofilm, are less sensitive to antimicrobial agents and the ability of bacteria in the biofilm to produce neutralizing agents that protects neighboring organisms [3]. Thus, studies have described an increase in resistance to antibiotics, due to their inadequate or excessive use [6, 7], as well as difficulties concerning the access of topical agents with effectiveness against the biofilm [8].

Chlorhexidine is a cationic broad-spectrum antimicrobial agent that has been widely studied and proven effective at controlling dental biofilm [9]. This effectiveness is directly related to a property denominated substantivity, by which 
the molecule remains adhered to tissues and has antibacterial action for up to 12 hours [10]. However, side effects lasting for more than 14 days are associated with chlorhexidine, such as pigmentation of the teeth and mucosa, an increase in the formation of supragingival calculus, a temporary loss of the sense of taste, a burning sensation, and dry mouth [9].

Antimicrobial photodynamic therapy (PDT) has emerged as an alternative to antibiotics for the treatment of microbial infections [6]. With this method, a photosensitizing agent is activated by light at a specific wavelength that corresponds to maximum absorbance by the substance, resulting in the production of free radicals, singlet oxygen, and other reactive oxygen species, which have a toxic effect on bacterial cells, leading to cell death without causing harm to the host $[6,7,11-13]$. This minimally invasive method is effective against resistant bacteria [14], has a rapid effect on the target organisms $[15,16]$, and does not lead to the development of resistance mechanisms $[6,17]$. Moreover, antimicrobial PDT is selective and painless and does not affect the patient's sense of taste [18].

Most oral bacteria do not absorb visible light from some type of low-power laser light. Therefore, nontoxic optical agent absorption used to be fixed at the bacterial walls, attracting to itself the laser at the moment of irradiation. It is essential to have antimicrobial action. Reactive oxygen species released by the association between the dye and the light source causes damage to various cellular structures, but primarily to DNA and cytoplasmic membrane [12], which affects differently gram-positive and gram-negative bacterias [34]. The cellular destruction depends on the association between the dye and the light source $[7,17]$. The effect of the dye is influenced of the kind, dose and site application. The efficacy of light can be influenced by wavelength, power density, energy fluence and the amount of oxygen available for the combination of both (dye and light) [11].

Different types of photosensitizers and light sources under various conditions have been used for the realization of photodynamic antimicrobial therapy [13]. For its use, a photosensitizer should have photophysical, chemical, and biological characteristics appropriate [11] among which is the ability to become an active drug and provide singlet oxygen, a broad spectrum of action, and affinity for microorganisms; low affinity for host cells promotes low mutagenicity and cytotoxicity associated with low possibility of developing resistant strains of microorganisms [11]. The light source, to be adequate, must present low power situated in the visible portion of the electromagnetic spectrum and specific wavelength resonant to dye. The wavelength depends on the dose and the depth of action of the photosensitizer used [11].

However, bacteria in biofilm have been demonstrated to be less affected by PDT than those in the plankton phase [35]. While a number of authors working with different light sources and photosensitizing agents report the efficacy of PDT in controlling dental biofilm by reducing the bacteria viability $[7,13,14,16,22-24,36-38]$, there is a lack of scientific evidence regarding the actual effectiveness of this method. Thus, the aim of the present study was to perform a systematic review of the literature on the efficacy of antimicrobial PDT on dental biofilm.

\section{Methods}

Articles addressing the effect of antimicrobial PDT with the use of a photosensitizing agent on known cariogenic biofilm formed mainly by streptococci of the mutans group and/or lactobacilli were included, with no restrictions placed on the method employed or year of publication. Reviews of the literature, studies involving only bacteria in the plankton phase, and studies involving an animal model were excluded.

2.1. Search Strategy. Searches were made of the Pubmed, Web of Science, Scopus, Lilacs, and Cochrane Library databases in October and November 2013 as well as March 2014. A manual search of the references of each article retrieved was also performed in an attempt to find further articles that were not in the electronic databases. Each database was searched from its inception to March 2014. The search was performed by two researchers and limited to studies involving human subjects published in the English language, using the following keywords: ("Biofilms"[Mesh] OR "Dental Plaque"[Mesh]) AND (photodynamic therapy OR antimicrobial photodynamic therapy OR light therapy).

2.2. Data Extraction and Evaluation of Methodological Quality. A total of 23 articles were retrieved from the databases and four additional articles were retrieved from the manual search of the reference lists. Following the reading of the title and abstract of each article, two independent raters (GCS and DSBO) selected studies for the full-text analysis. Interexaminer agreement was $96 \%$. Thirty-two articles were selected for the full-text analysis due to insufficient information in the abstract to support the decision regarding eligibility. Articles that did not address antimicrobial PDT, those that did not involve potentially cariogenic biofilm, those that used microorganisms in the plankton phase, and reviews of the literature were excluded. After the full-text analyses, seventeen articles were included in the present systematic review (Figure 1).

Data extraction and the evaluation of methodological quality were performed by two independent raters (GCS and DSBO). The evaluation involved the use of a chart considering the sample (sample size calculation $=1$; randomization $=$ 1 ), study design (in vivo $=3$; in situ $=2$; in vitro $=1$ ), control group (present $=1$; absent $=0$ ), blinding (doubleblind $=2$; single-blind; absent $=0$ ), and repetition of the experiment (yes $=1$; no $=0$ ). The maximum score was 9 points. Disagreements between the raters were discussed and resolved by consensus. The determination and critical analysis of the quality of the articles allowed suggestions for improvements in future studies.

\section{Results}

Among the total of 240 articles retrieved during the original search of the databases and references lists, seventeen were selected for the present systematic review for addressing the efficacy of antimicrobial PDT on biofilm with cariogenic potential. All seventeen articles described either in vitro or in situ studies. Table 2 offers a summary of the findings. 


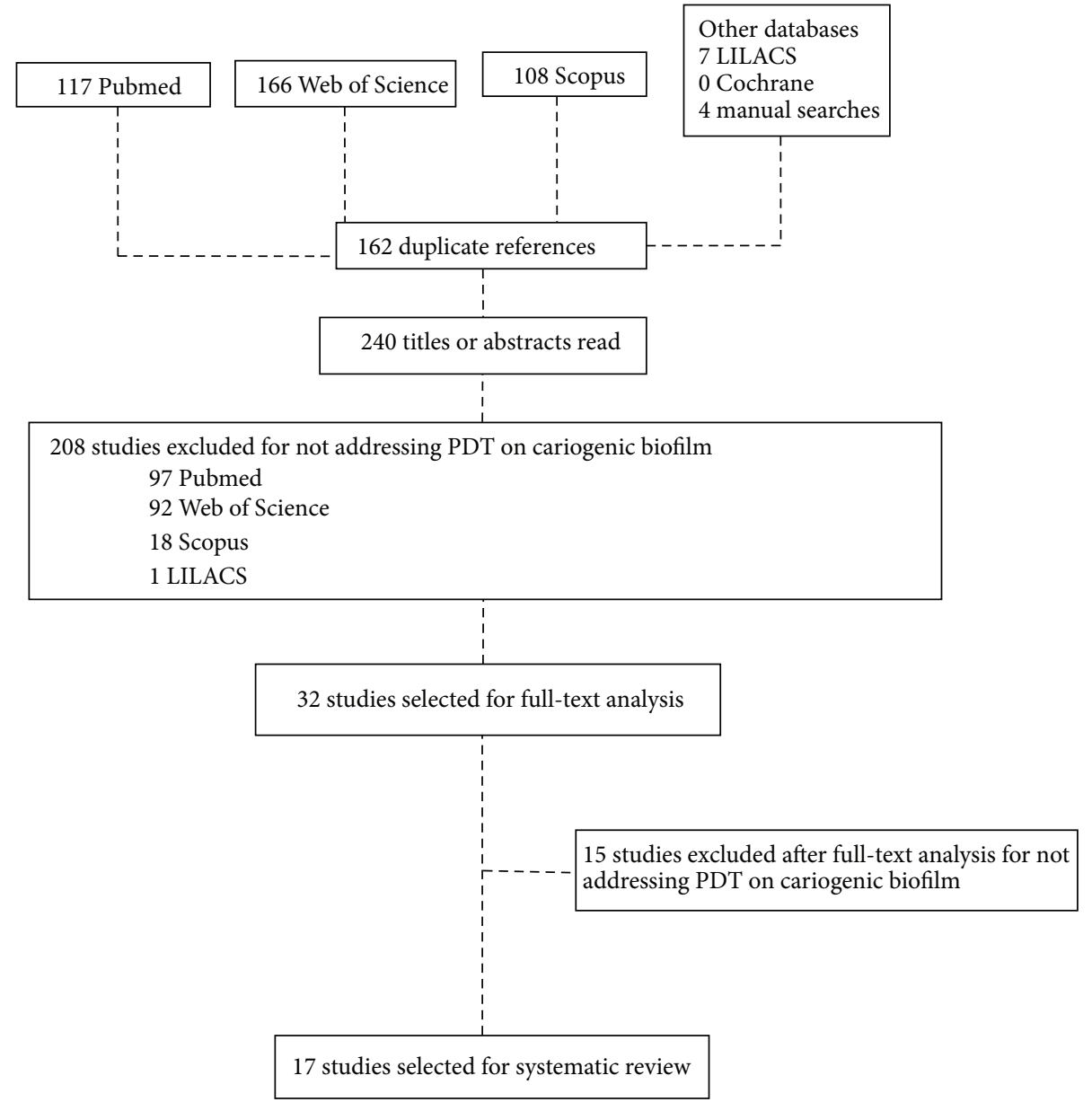

FIGURE 1: Flowchart demonstrating selection process of studies on PDT and dental biofilm.

Considerable variability among the articles was found regarding the photosensitizing agent. Toluidine blue was the most commonly employed. Each study used a specific light source (LED, laser, and light bulb), power, and application protocol.

Biofilm was cultivated in human saliva in three studies $[14,22,32]$, natural human biofilm was used in four studies $[19,21,27,30]$, and synthesized biofilm was used in ten studies $[16,20,23-26,28,29,31,33]$.

Two articles reported unfavorable results regarding the reduction of microorganisms in dental biofilm with the use of antimicrobial PDT $[25,30]$.

In the analysis of methodological quality, scores ranged from 1 to 5 points. The main drawbacks were related to the sample size calculation, randomization of the sample, blinding, and repetition of the experiment (Table 1).

\section{Discussion}

The analysis of the articles revealed the frequent lack of randomization of the specimens studied and failure to calculate the appropriate sample size. These data indicate possible selection bias. Moreover, divergent effects may have derived from systemic alterations in the different specimens and there is no possibility of reproducing the studies.

The majority of articles evaluated antimicrobial PDT on dental biofilm using an in vitro study, which is not the best design for arriving at adequate scientific evidence, although this model has led to significant advances in the study of dental biofilm [39]. In vitro models tend to involve a small number of species of microorganisms and laboratory conditions that may not adequately reflect the physiological situation in the oral cavity [40]. Factors such as salivary flow, the capacity of antimicrobial substances to adhere to the film on the teeth or the surface of soft tissues, and the interaction of noncultivatable bacteria cannot be modeled in an in vitro experiment [41].

The presence of polymeric extracellular substances, composition of the cell wall, growth rate, metabolic activity, and gene expression offer natural biofilm protection from the action of antimicrobial agents [42]. Moreover, nutritional status, temperature, $\mathrm{pH}$, and undereffective exposure to antimicrobial agents can enhance bacterial resistance to this type of treatment $[4,43]$. As biofilm is dependent on a number of factors, the use of a synthesized biofilm may not demonstrate the same scientific evidence as natural biofilm. 
TABLE 1: Quality scores of articles selected based on proposed evaluation scale.

\begin{tabular}{|c|c|c|c|c|c|c|}
\hline Authors and year & Sample & Study design & Control group & Blinding & Repetition of experiment & Total \\
\hline Wilson et al., 1995 [19] & 0 & 1 & 1 & 0 & 0 & 2 \\
\hline Wilson et al., 1996 [20] & 0 & 1 & 0 & 0 & 0 & 1 \\
\hline Wood et al., 1999 [21] & 0 & 2 & 1 & 0 & 0 & 3 \\
\hline O’Neill et al., 2002 [22] & 0 & 1 & 1 & 0 & 0 & 2 \\
\hline Zanin et al., 2005 [16] & 0 & 1 & 1 & 0 & 0 & 2 \\
\hline Wood et al., 2006 [23] & 0 & 1 & 1 & 0 & 1 & 3 \\
\hline Zanin et al., 2006 [24] & 1 & 1 & 1 & 0 & 0 & 3 \\
\hline Müller et al., 2007 [25] & 0 & 1 & 1 & 0 & 1 & 3 \\
\hline Steinberg et al., 2008 [26] & 0 & 1 & 1 & 0 & 1 & 3 \\
\hline Lima et al., 2009 [27] & 1 & 1 & 1 & 1 & 0 & 4 \\
\hline Schneider et al., 2012 [14] & 0 & 1 & 1 & 0 & 0 & 2 \\
\hline Chen et al., 2012 [28] & 0 & 1 & 1 & 0 & 0 & 2 \\
\hline Silva et al., 2012 [29] & 0 & 1 & 1 & 0 & 1 & 3 \\
\hline Teixeira et al., 2012 [30] & 1 & 2 & 1 & 1 & 0 & 5 \\
\hline Pereira et al., 2013 [31] & 0 & 1 & 1 & 0 & 0 & 2 \\
\hline Al-Ahmad et al., 2013 [32] & 0 & 2 & 1 & 0 & 1 & 4 \\
\hline Araújo et al., 2014 [33] & 0 & 1 & 1 & 0 & 1 & 3 \\
\hline
\end{tabular}

Dental biofilm has an organized structure formed by different types of microorganisms, which give the substance a complex, protective trait. Thus, studies employing biofilm composed of a single genus of microorganisms $[14,16,19,20$, $22-24,26,28,29,31]$ may not demonstrate the actual effect of antimicrobial PDT on dental biofilm in the oral cavity.

Although the majority of studies report favorable results with the use of antimicrobial PDT to reduce the volume of cariogenic microorganisms in the oral cavity, the articles offered a considerable variety of photosensitizing agents, light sources, application protocols, and methods for evaluating the effectiveness of the technique. This hinders the comparison of the findings, the reproducibility of events, and the determination of possible causality between the reduction in microorganisms and antimicrobial PDT. Moreover, the variations among the methods employed hamper the establishment of a possible protocol for the application of antimicrobial PDT on cariogenic biofilm.

Most of the articles included in this review used as photosensitizing dyes phenothiazine (methylene blue and toluidine blue). The physicochemical properties of the photosensitizers are important to the efficacy of photodynamic therapy. The ability of a component to absorb incident light does not mean it can act as a photosensitizer. Other requirements are important, such as having no toxic characteristics to the host cell, presenting toxicity only after activation by light, staying excited long enough to allow its interaction with neighboring molecules, producing cytotoxic species capable of causing bacterial killing time, and having high solubility in water $[8,44,45]$.

In oral antimicrobial photodynamic therapy, toluidine blue and methylene blue photosensitizing agents are the most commonly used [11, 12], since they have a high degree of selectivity for damage for gram-positive and gram-negative bacterias [46-48]. What determines the selectivity of this type of dye microbial cells is the interaction between the positive charges of the dye and the negative charges of the outer surface of the microbial cell [49]. The dye methylene blue is a prototype of phenothiazine derivatives and their use is attested almost a century and its relatively low toxicity to humans [50]. However, Wood et al. 2006 [23] noted the erythrosine better efficiency when compared to methylene blue and Photofrin on Streptococcus mutans biofilm.

The concentration of photosensitizers is still controversial. Al-Ahmad et al. 2013 [32] using different concentrations of toluidine blue $\left(5,10,25\right.$, and $\left.50 \mathrm{mg} / \mathrm{mL}^{-1}\right)$ found that the antimicrobial effect can be observed at lower concentrations.

The first light sources used in photodynamic therapy were conventional lamps with noncoherent, polychromatic light and a strong thermal component. With the development of lasers, which have particular characteristics, such monochromaticity, coherence, and collimation, the light source proved to be more efficient to photodynamic therapy. Diode lasers have resonant wavelength absorption band of most currently used dyes, act continuously, and are less portable and low in cost [51]. Currently, the light of a specific wavelength, sources which are most commonly applied in PDT are heliumneon $(\mathrm{HeNe})$ lasers, $(633 \mathrm{~nm})$ gallium-aluminum-arsenide (GaAlAs) diode lasers $(630-690,830$, or $906 \mathrm{~nm}$ ), and argon lasers $(488-514 \mathrm{~nm})$.

In this review, the majority of included studies used $\mathrm{HeNe}$ laser and LED (light emitting diode). LEDs are another alternative source of laser light and differ by presenting divergent beam, low thermal component and monochromatic light [51], and low cost [16]. Additionally, LED sources are present in the dental routine and can be used in PDT without requiring the acquisition of new equipment. However, no difference regarding the efficacy of these two types of light source for photodynamic therapy was observed [16]. 


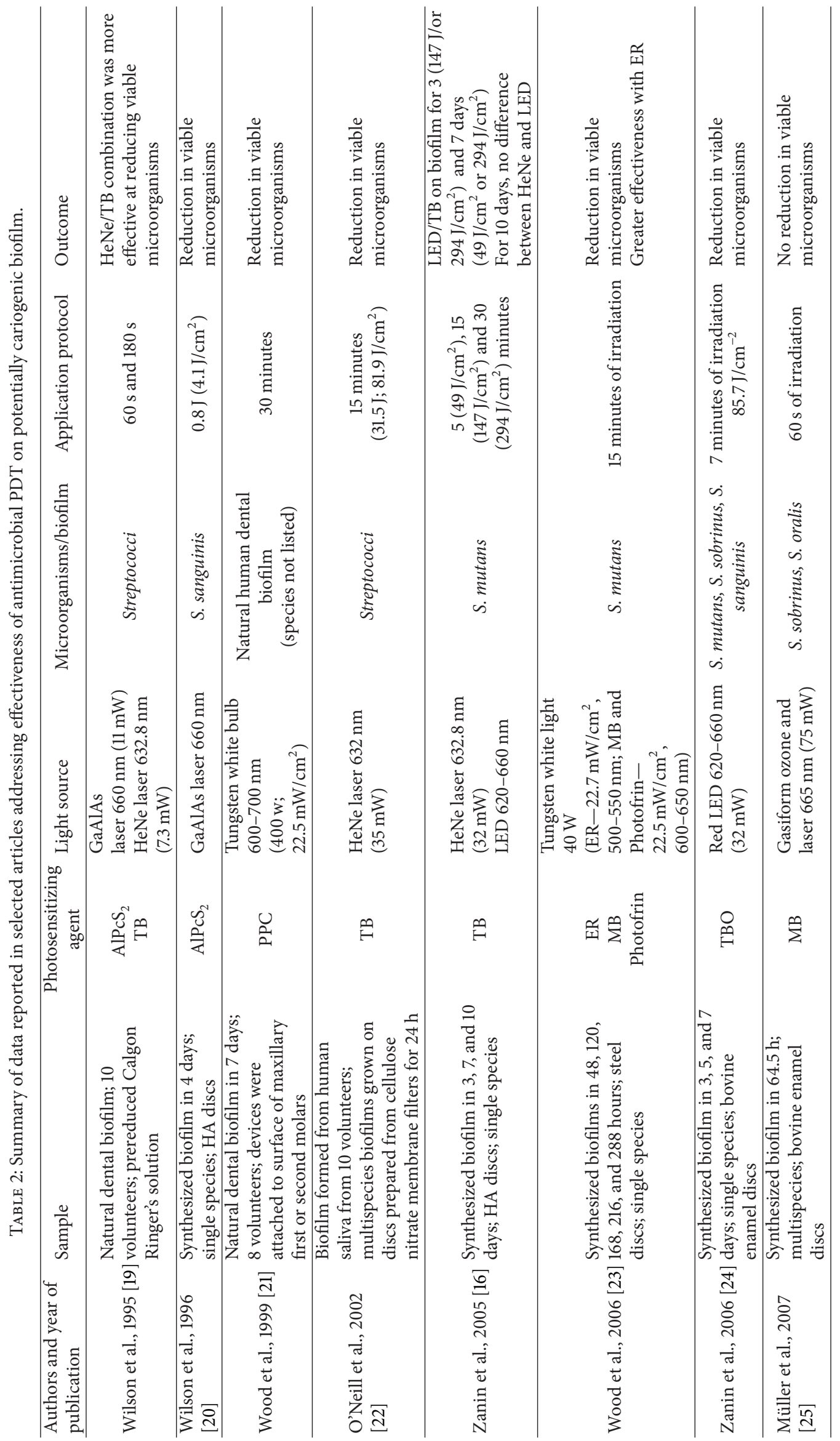




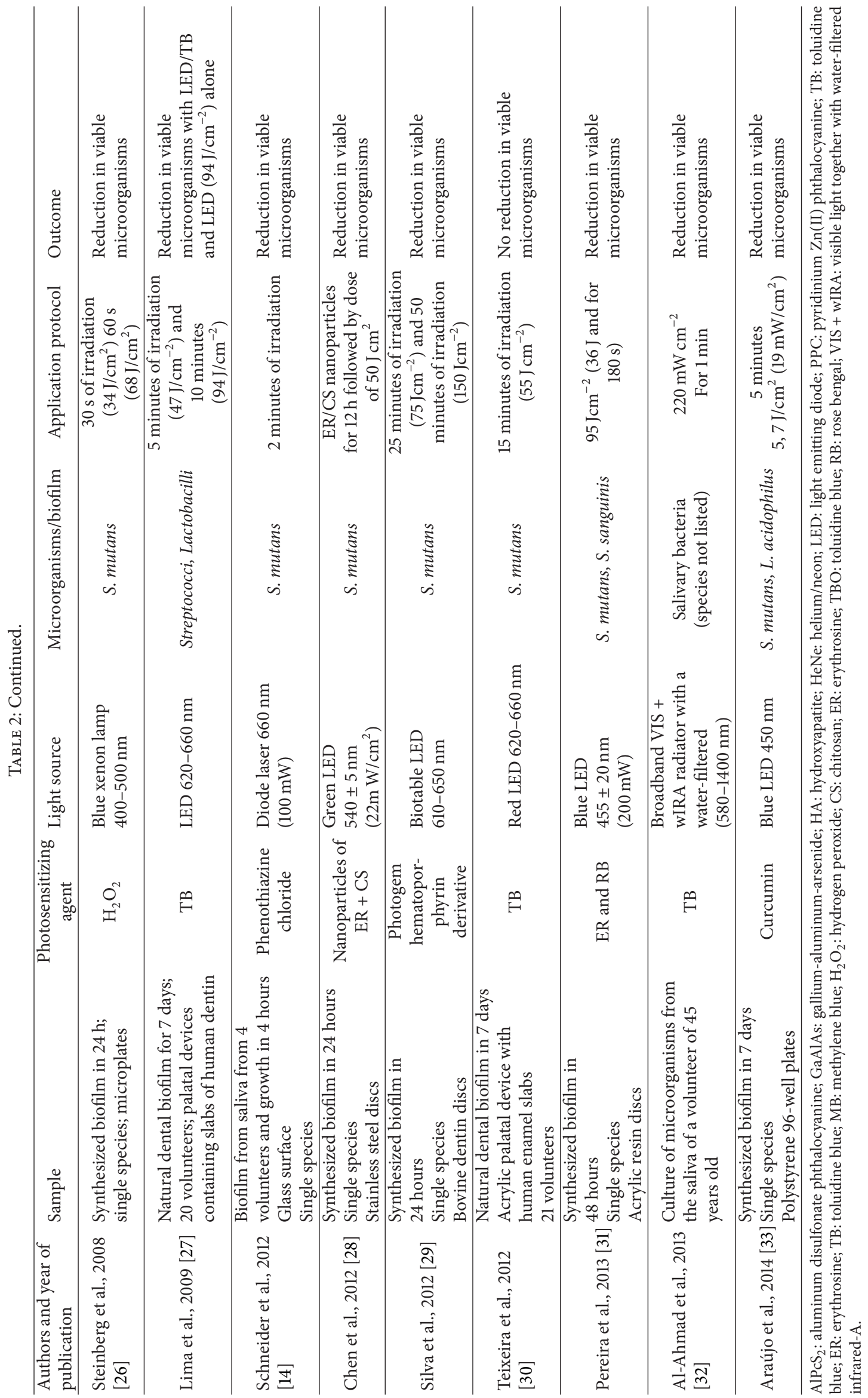


Considering the wavelength, blue light has been shown to be more efficient to be used in conjunction with a dye than red light $[52,53]$. However, the use of different types of light sources by authors evaluated in this review shows that there is no consensus regarding the type of light and the parameters to be used permanently in photodynamic therapy to control biofilm.

Two studies reported unfavorable results regarding the effect of antimicrobial PDT on dental biofilm [25, 30]. The authors attribute these findings to an increase in resistance to this technique among bacteria in biofilm and/or multispecies biofilm $[25,30]$ as well as the thickness [30] and age of the biofilm [25]. Similarly, resistance to antiseptics, such as chlorhexidine, has been described [54]. Moreover, antimicrobial efficacy is believed to be dose dependent [55], which would explain the lack of a positive effect, as antimicrobial PDT was only applied once in both studies [30]. The difficulty for antimicrobial agents, photosensitizing agents, and light to penetrate the deeper layers of biofilm limits their effectiveness $[25,30]$. However, studies have demonstrated that although antimicrobial PDT is threefold to fourfold less effective on thick, multispecies biofilm, antibiotics are as much as 250fold less effective [56]. There are alternatives that can enhance the effectiveness of antimicrobial PDT, such as the selection of photosensitizing agents capable of penetrating the matrix of the biofilm, the use of photomechanical waves to optimize the penetration of the photosensitizing agent [57], and internal irradiation of the biofilm using an optic fiber [22].

Some of the included studies investigated alternatives to optimize antimicrobial photodynamic therapy when applied in biofilm. The use of the visible light in combination with water-filtered infrared-A (VIS + wIRA) [32] showed satisfactory results, while the use of ozone gasiform [25] was not effective. The use of curcumin in biofilm decreased from 95 to $99.9 \%$ of viable microorganisms, depending on the concentration of the photosensitizing agent; however, when applied to carious dentin, their effectiveness was probably reduced by the difficulty of penetration of the photosensitizing agent [33].

Suggestions for Future Research. Despite the number of studies on antimicrobial PDT, greater knowledge is needed regarding the effectiveness of this form of treatment. Studies with methodological standardization, randomization, an adequate sample size, reproducibility, and adequate data analysis are needed. Moreover, the effectiveness of antimicrobial PDT on multispecies biofilm under real conditions, such as in an in situ and in vivo design, is needed to gain a better understanding of the action mechanism of this treatment modality and the determination of a possible application protocol.

\section{Conclusion}

The present systematic review of the literature does not allow drawing any concrete conclusions regarding the efficacy of antimicrobial PDT due to the contradictory findings and methodological differences. Although this method seems to be a promising option for reducing the quantity of cariogenic microorganisms in dental biofilm, there is no sufficiently strong scientific evidence to support this association.

Further experimental studies with methodological standardization, the use of natural human biofilm, and an in vivo design are needed to gain a better understanding of the mechanisms, indications, and possible side effects of antimicrobial photodynamic therapy.

\section{Conflict of Interests}

The authors declare that there is no conflict of interests regarding the publication of this paper.

\section{Authors' Contribution}

Ms. G. C. Santin contributed in the systematic review, analysis of studies, and paper writing. Ms. D. S. B. Oliveira contributed in the systematic review, analysis of studies, and paper writing. R. Galo contributed in the analysis of studies and paper writing. M. C. Borsatto contributed in paper writing. S. A. M. Corona contributed in paper writing.

\section{Acknowledgment}

The authors would like to thank Sandra Maria Tobias for being the language reviser of this paper.

\section{References}

[1] G. H. Dibdin and R. P. Shellis, "Physical and biochemical studies of Streptococcus mutans sediments suggest new factors linking the cariogenicity of plaque with its extracellular polysaccharide content.," Journal of Dental Research, vol. 67, no. 6, pp. 890-895, 1988.

[2] M. Shemesh, A. Tam, M. Feldman, and D. Steinberg, "Differential expression profiles of Streptococcus mutans ftf, gtf and vicR genes in the presence of dietary carbohydrates at early and late exponential growth phases," Carbohydrate Research, vol. 341, no. 12, pp. 2090-2097, 2006.

[3] P.D. Marsh, A. Moter, and D. A. Devine, "Dental plaque biofilms: communities, conflict and control," Periodontology 2000, vol. 55, no. 1, pp. 16-35, 2011.

[4] S. S. Socransky and A. D. Haffajee, "Dental biofilms: difficult therapeutic targets," Periodontology 2000, vol. 28, no. 1, pp. 1255, 2002.

[5] S. Reese and B. Guggenheim, "A novel TEM contrasting technique for extracellular polysaccharides in in vitro biofilms," $M i$ croscopy Research and Technique, vol. 70, no. 9, pp. 816-822, 2007.

[6] A. Tavares, C. M. B. Carvalho, M. A. Faustino et al., "Antimicrobial photodynamic therapy: study of bacterial recovery viability and potential development of resistance after treatment," Marine Drugs, vol. 8, no. 1, pp. 91-105, 2010.

[7] R. C. de Goulart, M. Bolean, T. P. de Paulino et al., "Photodynamic therapy in planktonic and biofilm cultures of Aggregatibacter actinomycetemcomitans," Photomedicine and Laser Surgery, vol. 28, no. 1, pp. 53-60, 2010.

[8] K. Konopka and T. Goslinski, "Photodynamic therapy in dentistry," Journal of Dental Research, vol. 86, no. 8, pp. 694-707, 2007. 
[9] E. Varoni, M. Tarce, G. Lodi, and A. Carrassi, "Chlorhexidine (CHX) in dentistry: state of the art," Minerva Stomatologica, vol. 61, no. 9, pp. 399-419, 2012.

[10] P. Bonesvoll, “Oral pharmacology of chlorhexidine," Journal of Clinical Periodontology, vol. 4, no. 5, pp. 49-65, 1977.

[11] S. Rajesh, E. Koshi, K. Philip, and A. Mohan, "Antimicrobial photodynamic therapy: an overview," Journal of Indian Society of Periodontology, vol. 15, no. 4, pp. 323-327, 2011.

[12] N. S. Soukos and J. M. Goodson, "Photodynamic therapy in the control of oral biofilms," Periodontology 2000, vol. 55, no. 1, pp. 143-166, 2011.

[13] T. S. Mang, D. P. Tayal, and R. Baier, "Photodynamic therapy as an alternative treatment for disinfection of bacteria in oral biofilms," Lasers in Surgery and Medicine, vol. 44, no. 7, pp. 588596, 2012.

[14] M. Schneider, G. Kirfel, M. Berthold, M. Frentzen, F. Krause, and A. Braun, "The impact of antimicrobial photodynamic therapy in an artificial biofilm model," Lasers in Medical Science, vol. 27, no. 3, pp. 615-620, 2012.

[15] M. Wilson, "Lethal photosensitisation of oral bacteria and its potential application in the photodynamic therapy of oral infections," Photochemical and Photobiological Sciences, vol. 3, no. 5, pp. 412-418, 2004.

[16] I. C. J. Zanin, R. B. Gonçalves, A. B. Junior, C. K. Hope, and J. Pratten, "Susceptibility of Streptococcus mutans biofilms to photodynamic therapy: an in vitro study," The Journal of Antimicrobial Chemotherapy, vol. 56, no. 2, pp. 324-330, 2005.

[17] C. N. Street, L. A. Pedigo, and N. G. Loebel, "Energy dose parameters affect antimicrobial photodynamic therapy-mediated eradication of periopathogenic biofilm and planktonic cultures," Photomedicine and laser surgery, vol. 28, supplement 1, pp. S61-66, 2010.

[18] N. S. Soukos, S. Som, A. D. Abernethy et al., "Phototargeting oral black-pigmented bacteria," Antimicrobial Agents and Chemoterapy, vol. 49, no. 4, pp. 1391-1396, 2005.

[19] M. Wilson, T. Burns, J. Pratten, and G. J. Pearson, "Bacteria in supragingival plaque samples can be killed by low-power laser light in the presence of a photosensitizer," Journal of Applied Bacteriology, vol. 78, no. 5, pp. 569-574, 1995.

[20] M. Wilson, T. Burns, and J. Pratten, "Killing of Streptococcus sanguis in biofilms using a light-activated antimicrobial agent," Journal of Antimicrobial Chemotherapy, vol. 37, no. 2, pp. 377381, 1996.

[21] S. Wood, B. Nattress, J. Kirkham et al., "An in vitro study of the use of photodynamic therapy for the treatment of natural oral plaque biofilms formed in vivo," Journal of Photochemistry and Photobiology B: Biology, vol. 50, no. 1, pp. 1-7, 1999.

[22] J. F. O’Neill, C. K. Hope, and M. Wilson, "Oral bacteria in multispecies biofilms can be killed by red light in the presence of toluidine blue," Lasers in Surgery and Medicine, vol. 31, no. 2, pp. 86-90, 2002.

[23] S. Wood, D. Metcalf, D. Devine, and C. Robinson, "Erythrosine is a potential photosensitizer for the photodynamic therapy of oral plaque biofilms," The Journal of Antimicrobial Chemother$a p y$, vol. 57, no. 4, pp. 680-684, 2006.

[24] I. C. J. Zanin, M. M. Lobo, L. K. A. Rodrigues, L. A. F. Pimenta, J. F. Höfling, and R. B. Gonçalves, "Photosensitization of in vitro biofilms by toluidine blue $\mathrm{O}$ combined with a light-emitting diode," European Journal of Oral Sciences, vol. 114, no. 1, pp. 6469, 2006.
[25] P. Müller, B. Guggenheim, and P. R. Schmidlin, "Efficacy of gasiform ozone and photodynamic therapy on a multispecies oral biofilm in vitro," European Journal of Oral Sciences, vol. 115, no. 1, pp. 77-80, 2007.

[26] D. Steinberg, D. Moreinos, J. Featherstone, M. Shemesh, and O. Feuerstein, "Genetic and physiological effects of noncoherent visible light combined with hydrogen peroxide on Streptococcus mutans in biofilm," Antimicrobial Agents and Chemotherapy, vol. 52, no. 7, pp. 2626-2631, 2008.

[27] J. P. M. Lima, M. A. S. De Melo, F. M. C. Borges et al., "Evaluation of the antimicrobial effect of photodynamic antimicrobial therapy in an in situ model of dentine caries," European Journal of Oral Sciences, vol. 117, no. 5, pp. 568-574, 2009.

[28] C.-P. Chen, C.-T. Chen, and T. Tsai, "Chitosan nanoparticles for antimicrobial photodynamic inactivation: characterization and in vitro investigation," Photochemistry and Photobiology, vol. 88, no. 3, pp. 570-576, 2012.

[29] T. C. Silva, A. F. F. Pereira, R. A. M. Exterkate et al., "Application of an active attachment model as a high-throughput demineralization biofilm model," Journal of Dentistry, vol. 40, no. 1, pp. 41-47, 2012.

[30] A. H. Teixeira, E. S. Pereira, L. K. A. Rodrigues, D. Saxena, S. Duarte, and I. C. J. Zanin, "Effect of photodynamic antimicrobial chemotherapy on in vitro and in situ biofilms," Caries Research, vol. 46, no. 6, pp. 549-554, 2012.

[31] C. A. Pereira, A. C. B. P. Costa, C. M. Carreira, J. C. Junqueira, and A. O. C. Jorge, "Photodynamic inactivation of Streptococcus mutans and Streptococcus sanguinis biofilms in vitro," Lasers in Medical Science, vol. 28, no. 3, pp. 859-864, 2013.

[32] A. Al-Ahmad, C. Tennert, L. Karygianni, K. T. Wrbas, E. Hellwig, and M. J. Altenburger, "Antimicrobial photodynamic therapy using visible light plus water-filtered infrared-A (wIRA)," Journal of Medical Microbiology, vol. 62, no. 3, pp. 467-473, 2013.

[33] N. C. Araújo, C. R. Fontana, V. S. Bagnato, and M. E. M. Gerbi, "Photodynamic antimicrobial therapy of curcumin in biofilms and carious dentine," Lasers in Medical Science, vol. 29, no. 2, pp. 629-635, 2014

[34] T. Maisch, "Anti-microbial photodynamic therapy: useful in the future?" Lasers in Medical Science, vol. 22, no. 2, pp. 83-91, 2007.

[35] C. R. Fontana, A. D. Abernethy, S. Som et al., "The antibacterial effect of photodynamic therapy in dental plaque-derived biofilms," Journal of Periodontal Research, vol. 44, no. 6, pp. 751759, 2009.

[36] C. K. Hope and M. Wilson, "Induction of lethal photosensitization in biofilms using a confocal scanning laser as the excitation source," The Journal of Antimicrobial Chemotherapy, vol. 57, no. 6, pp. 1227-1230, 2006.

[37] D. Metcalf, C. Robinson, D. Devine, and S. R. Wood, "Enhancement of erythrosine-mediated photodynamic therapy of Streptococcus mutans biofilms by light fractionation," Journal of Antimicrobial Chemotherapy, vol. 58, no. 1, pp. 190-192, 2006.

[38] S. F. G. Vilela, J. C. Junqueira, J. O. Barbosa, M. Majewski, E. Munin, and A. O. C. Jorge, "Photodynamic inactivation of Staphylococcus aureus and Escherichia coli biofilms by malachite green and phenothiazine dyes: an in vitro study," Archives of Oral Biology, vol. 57, no. 6, pp. 704-710, 2012.

[39] R. J. Palmer Jr., "Supragingival and subgingival plaque: paradigm of biofilms," Compendium of Continuing Education in Dentistry, vol. 31, no. 2, pp. 104-106, 2010.

[40] T. M. Auschill, E. Hellwig, A. Sculean, N. Hein, and N. B. Arweiler, "Impact of the intraoral location on the rate of biofilm 
growth," Clinical Oral Investigations, vol. 8, no. 2, pp. 97-101, 2004.

[41] A. Al-Ahmad, A. Wunder, T. M. Auschill et al., "The in vivo dynamics of Streptococcus spp., Actinomyces naeslundii, Fusobacterium nucleatum and Veillonella spp. in dental plaque biofilm as analysed by five-colour multiplex fluorescence in situ hybridization," Journal of Medical Microbiology, vol. 56, no. 5, pp. 681-687, 2007.

[42] J. W. Costerton, P. S. Stewart, and E. P. Greenberg, "Bacterial biofilms: a common cause of persistent infections," Science, vol. 284, no. 5418, pp. 1318-1322, 1999.

[43] M. R. W. Brown, D. G. Allison, and P. Gilbert, "Resistance of bacterial biofilms to antibiotics: a growth-rate related effect?" The Journal of Antimicrobial Chemotherapy, vol. 22, no. 6, pp. 777-780, 1988.

[44] Y. N. Konan, R. Gurny, and E. Allémann, "State of the art in the delivery of photosensitizers for photodynamic therapy," Journal of Photochemistry and Photobiology B: Biology, vol. 66, no. 2, pp. 89-106, 2002.

[45] P. Meisel and T. Kocher, "Photodynamic therapy for periodontal diseases: state of the art," Journal of Photochemistry and Photobiology B: Biology, vol. 79, no. 2, pp. 159-170, 2005.

[46] M. Wilson, J. Dobson, and W. Harvey, "Sensitization of oral bacteria to killing by low-power laser radiation," Current Microbiology, vol. 25, no. 2, pp. 77-81, 1992.

[47] M. Wilson and J. Dobson, "Lethal photosensitization of oral anaerobic bacteria," Clinical Infectious Diseases, vol. 16, no. 4, pp. S414-S415, 1993.

[48] M. N. Usacheva, M. C. Teichert, and M. A. Biel, "Comparison of the methylene blue and toluidine blue photobactericidal efficacy against gram-positive and gram-negative microorganisms," Lasers in Surgery and Medicine, vol. 29, no. 2, pp. 165-173, 2001.

[49] F. Harris, L. K. Chatfield, and D. A. Phoenix, "Phenothiazinium based photosensitisers-photodynamic agents with a multiplicity of cellular targets and clinical applications," Current Drug Targets, vol. 6, no. 5, pp. 615-627, 2005.

[50] M. N. Usacheva, M. C. Teichert, and M. A. Biel, "The role of the methylene blue and toluidine blue monomers and dimers in the photoinactivation of bacteria," Journal of Photochemistry and Photobiology B: Biology, vol. 71, no. 1-3, pp. 87-98, 2003.

[51] R. Ackroyd, C. Kelty, N. Brown, and M. Reed, "The history of photodetection and photodynamic therapy," Photochemistry and Photobiology, vol. 74, no. 5, pp. 656-669, 2001.

[52] C. Chui, K. Hiratsuka, A. Aoki, Y. Takeuchi, Y. Abiko, and Y. Izumi, "Blue LED inhibits the growth of Porphyromonas gingivalis by suppressing the expression of genes associated with DNA replication and cell division," Lasers in Surgery and Medicine, vol. 44, no. 10, pp. 856-864, 2012.

[53] C. Chui, A. Aoki, Y. Takeuchi et al., "Antimicrobial effect of photodynamic therapy using high-power blue light-emitting diode and red-dye agent on Porphyromonas gingivalis," Journal of Periodontal Research, vol. 48, no. 6, pp. 696-705, 2013.

[54] T. A. Millward and M. Wilson, "The effect of chlorhexidine on Streptococcus sanguis biofilms," Microbios, vol. 58, no. 236-237, pp. 155-164, 1989.

[55] B. P. F. A. Gomes, C. C. R. Ferraz, M. E. Vianna, V. B. Berber, F. B. Teixeira, and F. J. Souza-Filho, "In vitro antimicrobial activity of several concentrations of sodium hypochlorite and chlorhexidine gluconate in the elimination of Enterococcus faecalis," International Endodontic Journal, vol. 34, no. 6, pp. 424-428, 2001.
[56] M. J. Sedlacek and C. Walker, "Antibiotic resistance in an in vitro subgingival biofilm model," Oral Microbiology and Immunology, vol. 22, no. 5, pp. 333-339, 2007.

[57] N. S. Soukos, S. E. Mulholland, S. S. Socransky, and A. G. Doukas, "Photodestruction of human dental plaque bacteria: enhancement of the photodynamic effect by photomechanical waves in an oral biofilm model," Lasers in Surgery and Medicine, vol. 33, no. 3, pp. 161-168, 2003. 


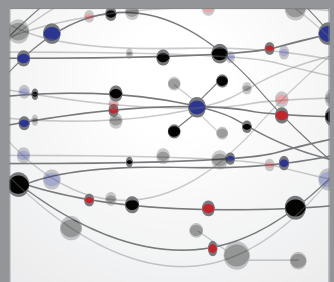

The Scientific World Journal
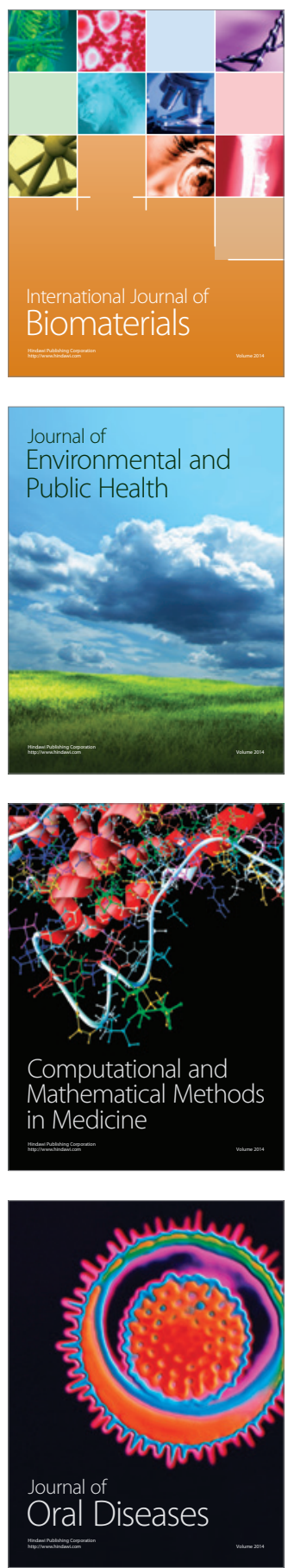
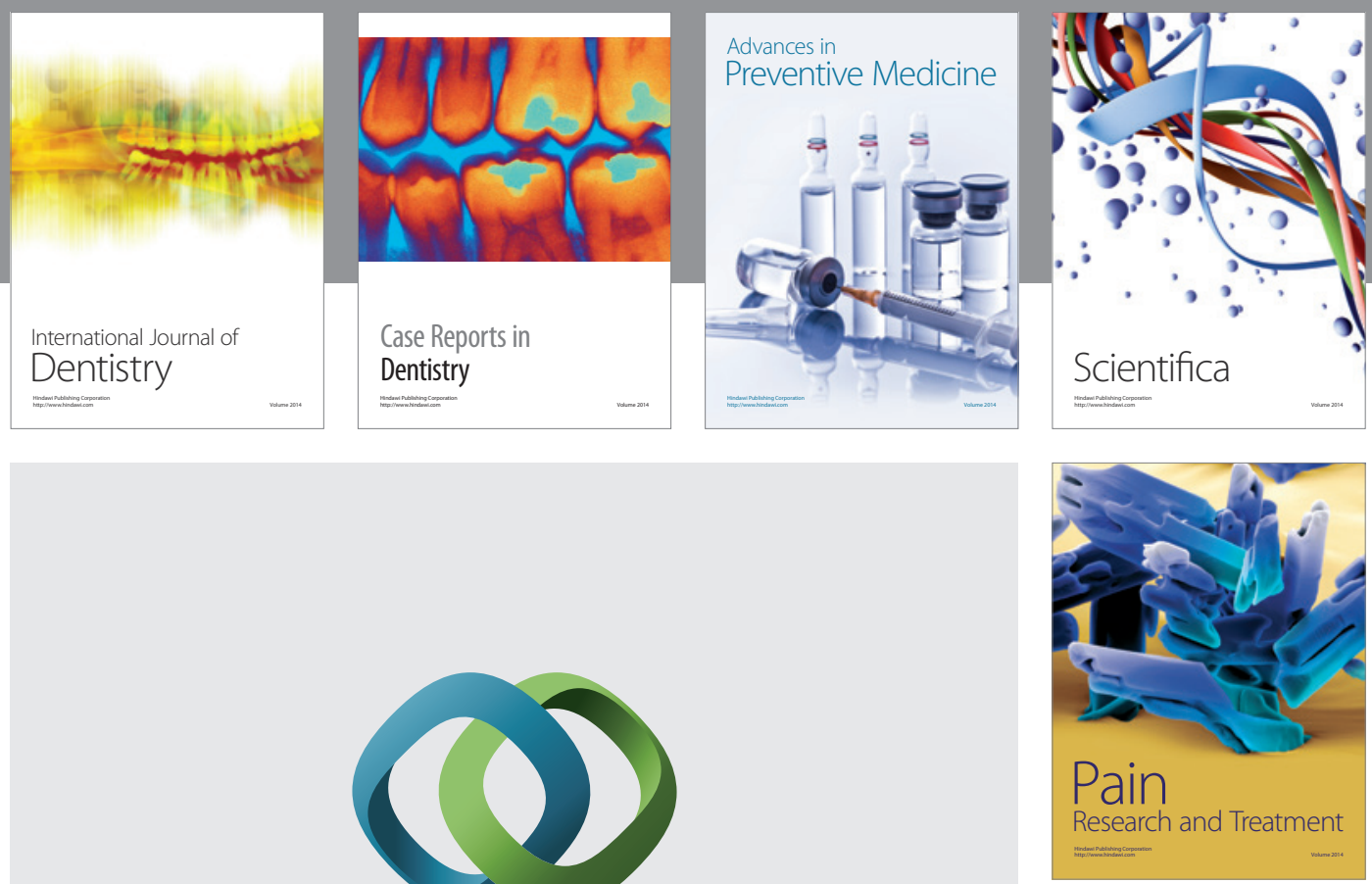

\section{Hindawi}

Submit your manuscripts at

http://www.hindawi.com
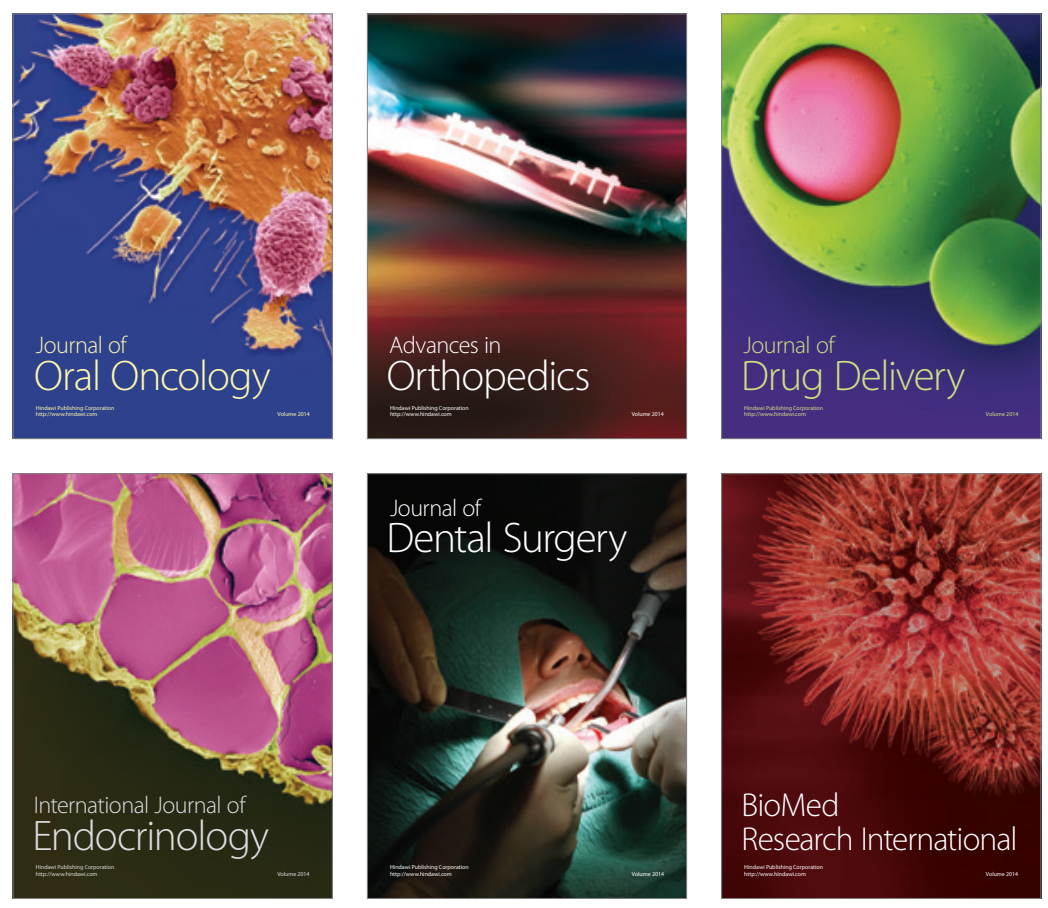

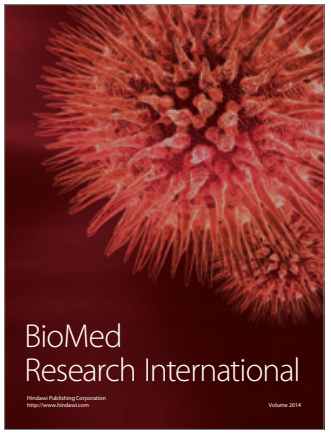

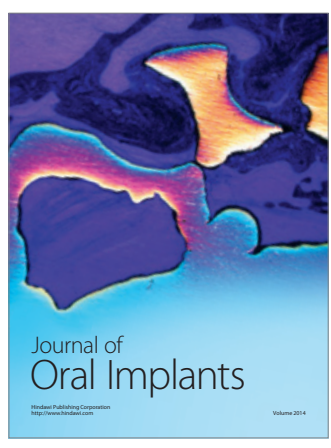
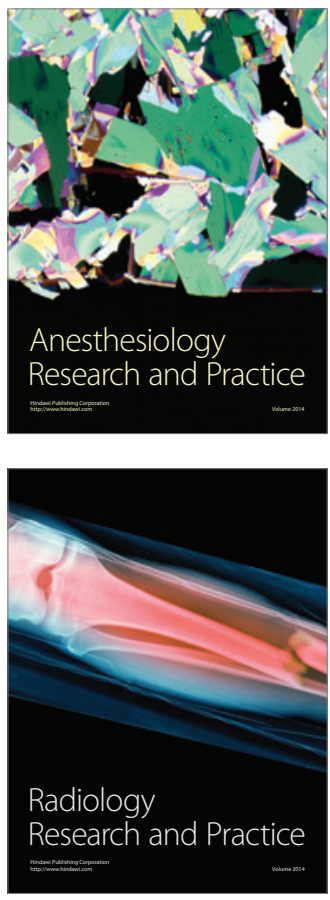